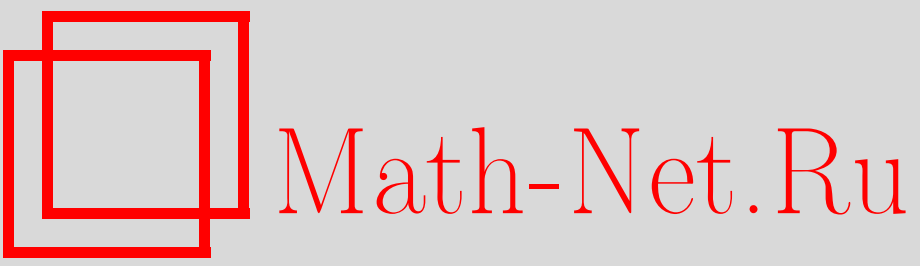

А. А. Владимиров, О накоплении собственных значений дифференциальных оператор-функций, УМH, 2002, том 57, выпуск 1, 151-152

DOI: https://doi.org/10.4213/rm480

Использование Общероссийского математического портала Math-Net.Ru подразумевает, что вы прочитали и согласны с пользовательским соглашением

http://www.mathnet.ru/rus/agreement

Параметры загрузки:

IP: 3.85 .5 .30

26 апреля 2023 г., 16:10:02 


\title{
О НАКОПЛЕНИИ СОБСТВЕННЫХ ЗНАЧЕНИЙ ДИФФЕРЕНЦИАЛЬНЫХ ОПЕРАТОР-ФУНКЦИЙ
}

\author{
А. А. ВлАдимиРов
}

Рассмотрим определенную на интервале $\Lambda=(\alpha, \beta) \subseteq \mathbb{R}$ оператор-функцию $L(\lambda)$, порожденную в $L_{2}[0,1]$ диффференциальным выражением

$$
l(\lambda)=(-1)^{n}\left(\frac{y^{(n)}(x)}{p_{0}(x, \lambda)}\right)^{(n)}+\sum_{k=1}^{n}(-1)^{n-k}\left(p_{k}(x, \lambda) y^{(n-k)}(x)\right)^{(n-k)}
$$

и самосопряженными краевыми условиями, которые запишем в матричном виде

$$
A(\lambda) \mathscr{Y}(y)+B(\lambda) \mathscr{Z}(y)=0 .
$$

Здесь $\mathscr{Y}(y), \mathscr{Z}(y)$ - векторы из $\mathbb{C}^{2 n}$ вида

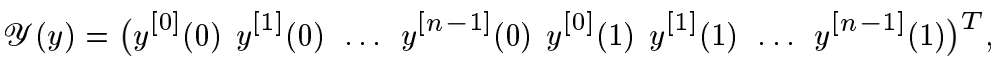

$$
\begin{aligned}
& \mathscr{Z}(y)=\left(y^{[n]}(0) \quad y^{[n+1]}(0) \quad \ldots \quad y^{[2 n-1]}(0) \quad y^{[n]}(1) \quad y^{[n+1]}(1) \quad \ldots \quad y^{[2 n-1]}(1)\right)^{T} \text {, }
\end{aligned}
$$

где квадратные скобки в показателе означают квазипроизводную (см. определение в $[1 ;$ гл. 5$]$ ), а $A(\lambda), B(\lambda)$ - матрицы размера $2 n \times 2 n$ такие, что ранг матрицы $(A(\lambda) \mid B(\lambda))$ равен $2 n$.

Следующие условия предполагаем в далшнейшем выполненными всюду, включая формулировки теорем: коэффициенты $p_{k}(x, \lambda)$ вешественны, суммируемы на $[0,1]$ и непрерывны по $\lambda$ как отображения из $\Lambda$ в $L_{1}[0,1]$; при любом $\lambda \in \Lambda$ неравенство $p_{0}(x, \lambda)>0$ выполнено для почти всех $x \in[0,1] ;$ элементы матриц $A(\lambda)$ и $B(\lambda)$ непрерывно зависят от $\lambda$; величина $\operatorname{rank} B(\lambda)$ постоянна на $\Lambda$. Существенность последнего условия показана в [2].

Цель заметки составляет ответ на вопрос, является ли один из концов интервала $\Lambda$ (например, $\beta$ ) точкой накопления спектра оператор-функция $L(\lambda)$.

Из теоремы 1 работы [2] вытекает следующий результат (через $\nu(S)$ здесь и далее обозначается кратность отрицательной части спектра оператора $S)$ :

Теорема 1. Пусть $\sigma_{L}(\Lambda)$ - спектр оператор-функции $L(\lambda)$ в интервале $\Lambda$ (он состоит из собственных значений $(C 3)$, возможнн, не изолированных). Тогда, если $\varlimsup_{\lambda \rightarrow \beta} \nu(L(\lambda))=+\infty$, то $\beta$ является предельной точкой множества $\sigma_{L}(\Lambda)$.

В теореме 2 работы [2] сформулированы условия, гарантирующие убьвание по $\lambda \in \Lambda$ собственных значений $\varkappa_{m}(\lambda)$ оператора $L(\lambda)$, занумерованных в порядке возрастания с учетом кратности. Далее эти условия будем называть условиями монотонности.

ТеОрема 2. При выполнении условий монотонности все CЗ оператор-функции $L(\lambda)$ изолировань, и $\beta$ является точкой накопления CЗ оператор-функции $L(\lambda)$ тогда и только тогда, когда $\overline{\lim }_{\lambda \rightarrow \beta} \nu(L(\lambda))=+\infty$.

Чтобы применять сформулированные общие теоремы при исследовании конкретных задач, надо иметь оценки кратности отрицательной части спектра операторов $L(\lambda)$. Для диффференциалных операторов второго порядка с разделенньми краевыми условиями подобные оценки были получены в [3]. Мы приведем соответствующие резултаты для простейших операторов четвертого порядка.

Пусть оператор-функция $L(\lambda)$ порождена выражением

$$
\left(\frac{y^{\prime \prime}(x)}{p(x, \lambda)}\right)^{\prime \prime}+q(x, \lambda) y(x)
$$

и краевыми условиями вида (2).

Работа вьполнена при поддержке Российского фонда фундаментальных исследований (гранты № 98-01-01000, 96-15-96091). 
Теорема 3. Пусть существует точка а $\in(0,1]$ такая, что функции $p(x, \lambda)$ и $q(x, \lambda)$ непрерывны как отображения из полуинтервала $(\alpha, \beta]$ в $L_{1, \operatorname{loc}}([0, a) \cup(a, 1])$, причем $p(x, \beta)>0$ почти всюду на $[0,1]$. Тогда если при некотором вещественном $\zeta$ существуют конечные предель

$$
p_{a}:=\lim _{x \rightarrow a} \frac{1}{p(x, \beta)|x-a|^{\zeta}}, \quad q_{a}:=\lim _{x \rightarrow a} \frac{q(x, \beta)}{|x-a|^{\zeta-4}}
$$

причем

$$
q_{a}<-\frac{1}{16}(\zeta-1)^{2}(\zeta-3)^{2} p_{a},
$$

то $\beta$ является точкой накопления СЗ оператор-функции $L(\lambda)$.

Если дополнительно выполнены условия монотонности, а неравенство (4) выполняется с заменой знака на противоположсный, то $\beta$ не является точкой накопления CЗ оператор-функции $L(\lambda)$.

В качестве примера приложения изложенных результатов к конкретной задаче рассмотрим оператор-функцию $L(\lambda)$, порожденную выражением

$$
\left(-(1+\lambda \alpha(x)) y^{\prime \prime}(x)\right)^{\prime \prime}-\left(\lambda \beta(x)+\lambda^{2} \rho(x)\right) y(x),
$$

где функции $\beta(x), \rho(x)$ и $\alpha(x)>0$ непрерьвны, и краевыми условиями

$$
y(0)=y^{\prime}(0)=y^{\prime \prime}(1)=y^{\prime \prime \prime}(1)=0 .
$$

Взяв за область значений спектрального параметра интервал $\left(-\infty,-\left(\alpha_{0}\right)^{-1}\right)$, где $\alpha_{0}:=$ $\min _{x \in[0,1]} \alpha(x)$, получим оператор-функцию рассматриваемого типа.

Предположим, что минимальное значение $\alpha_{0}$ достигается функцией $\alpha(x)$ в единственной, причем внутренней, точке $x_{0} \in[0,1]$. Применяя теорему 3, получаем следующий резултат:

ТеОрема 4. Пусть выполнено условие

$$
\alpha(x) \in C^{4}\left(x_{0}\right), \quad \alpha^{\prime \prime}\left(x_{0}\right)=0 .
$$

Тогда если

$$
\beta\left(x_{0}\right)-\frac{1}{\alpha_{0}} \rho\left(x_{0}\right)<-\frac{3}{128} \alpha^{(\mathrm{IV})}\left(x_{0}\right),
$$

то $-\left(\alpha_{0}\right)^{-1}$ является точкой накопления CЗ оператор-функции $L(\lambda)$.

Если выполнены условия $\rho(x) \leqslant 0$ и (7), а неравенство (8) выполнено с заменой знака неравенства на противоположный, то $-\left(\alpha_{0}\right)^{-1}$ не является точкой накопления СЗ оператор-функции $L(\lambda)$.

Автор благодарит А. А. Шкаликова за постановку задач и ценные замечания.

\section{СПИСОК ЛИТЕРАТУРЫ}

[1] М.А. Наймарк. Линейные диффференциальные операторы. М.: Наука, 1969. [2] А. А. Владимиров // Матем. заметки. 2000. Т. 68. № 3. C. 471-474. [3] R. Mennicken, H. Schmid, A. A. Shkalikov // Math. Nachr. 1998. V. 189. P. 157-170.

Принято редколлегией 26.12 .2001 